\title{
Desorption of vitamin E from silica-packed fixed-bed column by isopropanol.
}

\begin{abstract}
Desorption of vitamin E from silica-packed fixed-bed column was studied as functions of column bed height, column temperature and flow rate of isopropanol. Isopropanol was the desorbing solvent and it was eluted through the columns saturated with vitamin $\mathrm{E}$. The desorption profiles of all systems showed that vitamin $\mathrm{E}$ might desorb at two distinct rates simultaneously. The slow desorbing step was the rate-controlling process for recovery of vitamin E. The desorption rate increased with the decrease of column bed height and flow rate, but increased with increasing column temperature. This indicated that the desorption process was an endothermic process. The percentage recovery of vitamin E upon completion of desorption was considered high for all systems, ranging from 94.8 to $98.8 \%$, with vitamin E concentration in the extract of $18.5-21.5 \%$. Although the bed height, column temperature and flow rate were functions of desorption rate, it appeared that percentage recovery and vitamin $\mathrm{E}$ concentration in the extract were rather unaffected by the operating conditions tested if the column was eluted by isopropanol for a sufficient time to desorb vitamin $\mathrm{E}$. Nevertheless, the use of isopropanol would be more efficient if desorption was carried out at lower flow rate and higher column temperature.
\end{abstract}

Keyword: Recovery; Silica; Desorption. 